ఠ

\title{
Peptide receptor radionuclide therapy in the management of gastrointestinal neuroendocrine tumors: efficacy profile, safety, and quality of life
}

This article was published in the following Dove Press journal:

OncoTargets and Therapy

27 January 2017

Number of times this article has been viewed

\author{
Stefano Severi' \\ Ilaria Grassi \\ Silvia Nicolini' \\ Maddalena Sansovini' \\ Alberto Bongiovanni² \\ Giovanni Paganelli' \\ 'Nuclear Medicine Unit, \\ ${ }^{2}$ Osteoncology and Rare Tumors \\ Center, Istituto Scientifico Romagnolo \\ per lo Studio e la Cura dei Tumori \\ (IRST) IRCCS, Meldola, Italy
}

\begin{abstract}
Peptide receptor radionuclide therapy (PRRT), developed over the last two decades, is carried out using radiopharmaceuticals such as 90Y-DOTA-Tyr3-octreotide and 177Lu-DOTATyr3-octreotate (177Lu-Dotatate). These radiocompounds are obtained by labeling a synthetic somatostatin analog with a $\beta$-emitting radioisotope. The compounds differ from each other in terms of their energetic features (due to the radionuclide) and peptide receptor affinity (due to the analog) but share the common characteristic of binding specific membrane somatostatin receptors that are (generally) overexpressed in neuroendocrine neoplasms (NENs) and their metastases. NENs are tumors arising from diffuse neuroendocrine system cells that are classified according to grading based on Ki67 percentage values (Grades 1 and 2 are classed as neuroendocrine tumors [NETs]) and to the anatomical site of occurrence (in this paper, we only deal with gastroenteropancreatic [GEP]-NETs, which account for $60 \%-70 \%$ of all NENs). They are also characterized by specific symptoms such as diarrhea and flushing (30\% of cases). Despite substantial experience gained in the area of PRRT and its demonstrable effects in terms of efficacy, safety, and improvement in quality of life, these compounds are still not registered (registration of $177 \mathrm{Lu}$-Dotatate for the treatment of midgut NETs is expected soon). Thus, PRRT can only be used in experimental protocols. We provide an overview of the work of leading groups with wide-ranging experience and continuity in data publication in the area of GEP-NET PRRT and report our own personal experience of using different dosage schedules based on the presence of kidney and bone marrow risk factors. Our results on the retreatment of patients previously administered 90Y-DOTA-Tyr3-octreotide with a low dosage of 177Lu-Dotatate are also included. A comment on potential future developments of PRRT in GEP-NETs is provided.
\end{abstract}

Keywords: 90Y-Dotatoc, 177Lu-Dotatate, radiopharmaceutical, radiolabelled receptors, neuroendocrine malignancies, delivered activity

\section{Introduction}

Neuroendocrine neoplasms (NENs) are tumors arising from diffuse neuroendocrine system cells and are characterized by high expression of somatostatin receptors (SSTRs) and specific markers, including CgA, synaptophysin, CD53 protein, and the neuron-specific enolase. Given the heterogeneity of these tumors, NEN classification is currently based on the following:

- Grading: leading to classification into well-differentiated tumors or Grade 1 (Ki67 <3\%), moderately differentiated tumor or Grade 2 (Ki67 between 3\% and $20 \%$ ), or poorly differentiated tumors or Grade 3 (Ki67 >20\%). Grade 1 and 2 tumors are grouped into NETs, while Grade 3 tumors are classed as neuroendocrine carcinomas. $^{1,2}$
Nuclear Medicine Unit, Istituto Scientifico

Romagnolo per lo Studio e la Cura dei

Tumori (IRST) IRCCS, via P. Maroncelli 40,

47014 Meldola (FC), Italy

Tel +390543739323

Fax +39054373932I

Email stefano.severi@irst.emr.it hY $\mathrm{NC}$ and incorporate the Creative Commons Attribution - Non Commercial (unported, v3.0) License (http://creativecommons.org//icenses/by-nc/3.0/). By accessing the work you
hereby accept the Terms. Non-commercial uses of the work are permitted without any further permission from Dove Medical Press Limited, provided the work is properly attributed. For permission for commercial use of this work, please see paragraphs 4.2 and 5 of our Terms (https://www.dovepress.com/terms.php). 
- Site of occurrence: although NETs may arise in any body region, $60 \%-70 \%$ originate in the gastroenteropancreaticNENs (GEP-NENs) tract.

NETs are also characterized by the presence of associated symptoms and as such are considered as functioning tumors (30\% of cases). Symptoms relating to hormonal hypersecretion frequently include diarrhea, flushing, right heart failure, and hyper- or hypoglycemia.

Peptide receptor radionuclide therapy (PRRT), developed over the last two decades, is based on the use of a receptor target and represents one of the most effective treatments for well-differentiated unresectable or metastatic NETs. This therapy is performed by labeling a synthetic and stable somatostatin analog with a $\beta$-emitting radioisotope. The analog binds specific membrane receptors that are generally overexpressed in NETs and their metastases. ${ }^{3}$ The complex formed is chemically stable and is internalized into the cell in a favorable position for the irradiation of the nucleus. In addition, the long half-life of the analog allows for a persistent therapeutic effect. The binding mechanism between the somatostatin analog and its receptors has also been used for diagnostic purposes, ie, in scintigraphic exams with 111 In and $68 \mathrm{Ga}$ peptide PET. ${ }^{4,5}$

Historically, the first therapeutic experiences in NETs were performed using $111 \mathrm{In}$-pentetreotide and produced a clinical benefit but rarely a radiological response. ${ }^{6}$ Subsequently, a high-energy $\beta$ emitter, yttrium-90, was evaluated (Emax $2.27 \mathrm{MeV}$, half-life 64 hours, tissue penetration $7 / 8 \mathrm{~mm}$ ). This radioisotope was linked to octreotide, constituting 90Y-DOTA-Tyr3-octreotide (90Y-Dotatoc), a highenergy radiopharmaceutical with good receptor affinity for the SSTR2 peptide. Studies on the use of 90Y-Dotatoc in clinical trials have reported objective response rates in $6 \%-37 \%$ of patients and have shown a positive impact on overall survival (OS), but these trials are highly heterogeneous..$^{7-9}$
In the mid-2000s, another radiopharmaceutical, 177LuDOTA-Tyr3-octreotate (177Lu-Dotatate), was synthesized. The lower tissue penetration of 177Lutetium (Emax 0:49 $\mathrm{MeV}$, Rmax $2 \mathrm{~mm}$ ) is balanced by a longer half-life ( 6.7 days) of the radionuclide and by the higher peptide receptor affinity (about ninefold higher) for SSTR2s, overexpressed in the majority of NETs. Furthermore, 177Lutetium has a significant $\gamma$ emission, which enables good quality posttreatment images to be acquired. These images provide an accurate representation of the distribution of the therapy between all the tumor lesions and can be used to monitor changes in the disease during treatment. The impact of $177 \mathrm{Lu}$-Dotatate PRRT on PFS has been evaluated in numerous papers, with a general consensus reached on its good tolerability, especially for the kidneys, and on its high efficacy. ${ }^{10}$ Results obtained in clinical trials with 90Y-Dotatoc and 177Lu-Dotatate have shown good activity and acceptable tolerability ${ }^{11}$ (Table 1).

Dosimetry studies have confirmed the high specificity of these therapies and their aggressiveness against tumors, together with their ability to spare healthy tissue. Current administration schedules comprise the most appropriate number of cycles (generally four or five) and the best delivery frequency ( $6 \pm 2$ weeks apart). This treatment periodicity allows the patient to recover from the mild side effects of the therapy and increases its effectiveness in terms of the radiobiological activity. ${ }^{12}$

PRRT has proven highly effective in reducing specific symptoms of the neuroendocrine syndrome (diarrhea, bothersome flushing of the face, and cardiac function disorders due to right ventricular dysfunction), which represent a major handicap. PRRT has also proven analgesic efficacy, especially against pain caused by bone metastases that frequently occur in intestinal and bronchial tumors. ${ }^{13}$ The therapy, albeit generally well-tolerated, is not completely free from side effects that can be classified as acute or delayed. ${ }^{14}$ The acute

Table I Summary of most relevant papers on PRRT in terms of center experience and number of patients treated

\begin{tabular}{|c|c|c|c|c|c|c|c|c|c|}
\hline Study & Ligand & $\begin{array}{l}\text { Number } \\
\text { of patients }\end{array}$ & $\begin{array}{l}\text { Tumor } \\
\text { origin }\end{array}$ & $\begin{array}{l}\text { Response } \\
\text { criteria }\end{array}$ & OR (\%) & SD (\%) & DCR & PFS/TTP & Outcome \\
\hline Imhof et al, $7201 \mathrm{I}$ & 90Y-Dotatoc & 1,109 & NETs & RECIST & 34 & 5.2 & 39 & $13 \mathrm{~m}$ & OS $23 \mathrm{~m}$ \\
\hline Bushnell et al, ${ }^{8} 2010$ & 90Y-Dotatoc & 90 & NETs & SWOG & 4 (PR) & 70 & 74 & $16 \mathrm{~m}$ & Median OS $26 \mathrm{~m}$ \\
\hline Kwekkeboom et al, ${ }^{21} 2008$ & I77Lu-Dotatate & 310 & GEP-NETs & SWOG & 30 & 51 & 19 & $33 \mathrm{~m}$ & Median OS $46 \mathrm{~m}^{\mathrm{a}}$ \\
\hline Ezziddin et al, ${ }^{22} 2014$ & I77Lu-Dotatate & 68 & PNETs & RECIST & 60 & 25 & 15 & $34 \mathrm{~m}$ & Median OS $53 \mathrm{~m}$ \\
\hline Garkavij et al, ${ }^{35} 2010$ & I77Lu-Dotatate & 12 & NETs & RECIST & $17(\mathrm{PR})$ & 57 & 74 & - & - \\
\hline Swärd et al, ${ }^{36} 2010$ & I77Lu-Dotatate & 26 & NETs & RECIST & 38 & 50 & 88 & - & - \\
\hline Bodei et al, ${ }^{37} 201 \mathrm{I}$ & I77Lu-Dotatate & 42 & NETs & RECIST & 31 & 47 & 78 & $36 \mathrm{~m}$ & OS $36 \mathrm{~m}$ \\
\hline
\end{tabular}

Note: a Median OS from start of treatment.

Abbreviations: OR, overall response; SD, stable disease; DCR, disease control rate; PFS, progression-free survival; TTP, time-to-progression; PR, partial response; OS, overall survival; m, months; GEP, gastroenteropancreatic; NETs, neuroendocrine tumors; PNETs, pancreatic NETs; RECIST, Response Evaluation Criteria in Solid Tumors; SWOG, Southwest Oncology Group; PRRT, peptide receptor radionuclide therapy; 90Y-Dotatoc, 90Y-DOTA-Tyr3-octreotide; I77Lu-Dotatate, I77Lu-DOTATyr3-octreotate. 
side effects, usually mild, are nausea, headache, and, rarely, vomiting; they occur more often in the early postinjection phase and are generally transient. PRRT may exacerbate the neuroendocrine syndrome which, when present, can be treated with symptomatic drugs. The most important delayed side effects are renal and bone marrow toxicity. Renal toxicity is generally avoided or limited by renal protection, which can be obtained with amino acid infusion (arginine or lysine) before and during PRRT. These amino acids, competing with the site of tubular reabsorption of the drug, reduce recirculation of the radioactivity inside the kidneys, thus limiting their exposure. Comparative studies have demonstrated that the competitive action of these amino acids in the kidney is capable of reducing exposure by $39 \%$ when coverage of the amino acid lasts for up to 10 hours after administration of the therapy, and by $65 \%$ when it lasts for 2 days. ${ }^{15,16}$ Despite substantial experience gained in this area, PRRT radiopharmaceuticals have never been registered and therapy is still only available through the use of experimental protocols. A 177Lu-Dotatate compound (Lutathera ${ }^{\circledR}$, Advanced Accelerator Applications, Saint-Genis-Pouilly, France), which has successfully been tested in midgut NETs, is currently being evaluated for registration.

Generally, patients included in experimental protocols have a positive NET histology and a positive SSTR scintigraphy with $111 \mathrm{In}$ OctreoScan or 68Ga PET-Dota-peptide. A scan is considered positive if lesion uptake is either equal to or greater than liver uptake. Inclusion criteria also generally include objective disease progression, normal blood count (especially adequate hemoglobin, white cell, and platelet count levels) creatinine, and Eastern Cooperative Oncology Group $\leq 2$. Furthermore, a good performance status guarantees patient self-sufficiency and reduces the need for physical assistance during the treatment period (3 days/cycle) spent in a protected hospital environment. Life expectancy is classically set at a minimum of 6 months.

Over time, the use of PRRT, which was created specifically for neuroendocrine tumors, has been extended to other types of confirmed SSTR-positive tumors. Thus, tumors such as non-iodine-absorbing differentiated thyroid cancer, breast cancer, lymphoma, glioma, meningioma, and paraganglioma can be treated with PRRT when registered therapies have failed.

\section{PPRT in GEP-NETs 90Y-Dotatoc}

In 2012, an overview on Yttrium-90 Dotatoc therapy in GEPNETs was published by the Milan group, ${ }^{9}$ in which tolerability, efficacy, and prospects were discussed. With regard to tolerability, acute side effects were usually mild: fatigue and endocrine syndrome exacerbation were rare and nausea (or more rarely vomiting) was frequently related to amino acid coinfusion. Kidney and bone marrow toxicity were the most important dose-limiting factors. The problem of finding the administered activity threshold was again processed and a role for bioeffective dose (BED) in predicting toxicity speculated. About 40 Gy of BED was indicated as a more likely threshold for renal toxicity by Dale ${ }^{17}$ and Barone. ${ }^{18}$ All of these authors suggested using a lower BED (28 Gy) threshold if risk factors (ie, long-standing and poorly controlled diabetes and hypertension) were present, and a higher BED (40 Gy) threshold if there were no risk factors. ${ }^{19}$ In the study by Bodei et al, ${ }^{19}$ the authors defined that the administered dosage and number of cycles administered can effectively contribute to reducing renal and bone marrow dosimetry. In addition, these authors argued that the possible coexistence of unconjugated radionuclides labeling bone in a nonspecific way could increase bone marrow toxicity. In a multicenter study, PRRT with $90 Y-D o t a t o c$ was shown to induce clinically stable disease (SD) or partial response (PR), according to Southwest Oncology Group (SWOG) criteria, in 74\% of patients, leading to a positive impact on survival. PFS was also significantly longer when symptoms such as diarrhea were reduced (Figure 1). ${ }^{8}$ In a Phase I/II study by the Kwekkeboom group, ${ }^{20} 58$ patients with GEP-NETs treated with 1.7-32.8 GBq of PRRT reported a clinical benefit in $57 \%$ of cases (the true objective response was $20 \%$ ), with a median OS of 36.7 months.

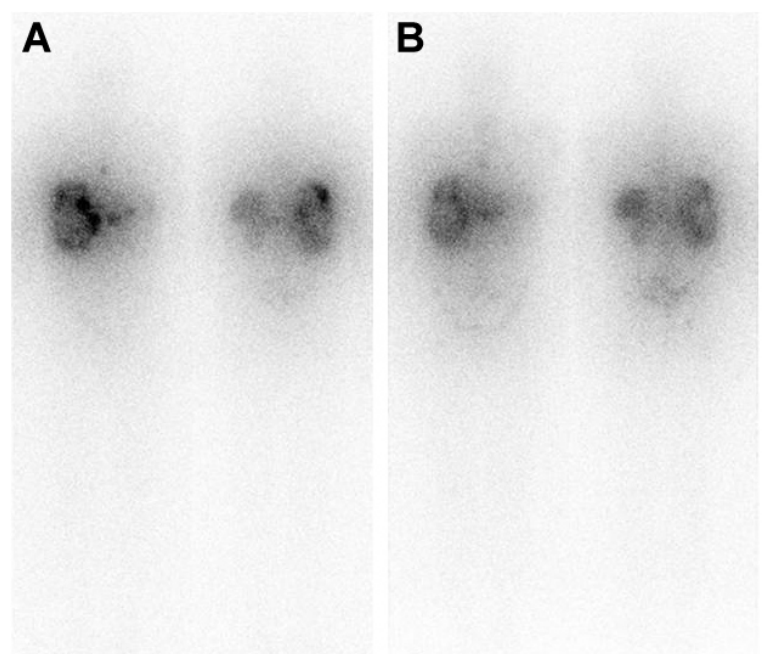

Figure I Patient with progressive GEP-NET treated at our institute.

Notes: Total body images acquired after the first (A) and fifth (B) cycle of $90 \mathrm{Y}$ Dotatoc. Cumulative activity delivered was $8.14 \mathrm{GBq}$.

Abbreviations: GEP, gastroenteropancreatic; NET, neuroendocrine tumor; $90 \mathrm{Y}$ Dotatoc, 90Y-DOTA-Tyr3-octreotide. 


\section{I77Lu-Dotatate/Dotatoc}

In 2008, the Rotterdam group ${ }^{21}$ was the first to report toxicity and efficacy data on 504 and 310 GEP-NETs patients, respectively, treated with $27.8-29.6 \mathrm{GBq}$ of $177 \mathrm{Lu}$-Dotatate over a mean of four cycles performed 6/10 weeks apart. Grade 3 or 4 hematological toxicity was present in $3.6 \%$ of administrations, with myelodysplastic syndrome in three cases and transient nonfatal liver toxicity in two cases. With regard to efficacy, a complete response was registered in 3\% of cases, PR in $28 \%$, and minor response (MR) in $16 \%$. The mean time to progression was 40 months and median OS from diagnosis was 128 months.

The Bonn and Frankfurt (Germany) groups ${ }^{22}$ retrospectively analyzed 74 metastatic and progressive GEP-NET patients treated with 177Lu-Dotatate PRRT using $7.9 \mathrm{GBq}$ per cycle over a mean four cycles treatment performed 3 months apart. The median follow-up period was 47 months and data were analyzed using SWOG criteria. Results showed $36.3 \% \mathrm{PR}, 17.6 \% \mathrm{MR}, 35.1 \% \mathrm{SD}$, and $10.8 \%$ progressive disease (PD). The median PFS was 26 months from the start of treatment and the median OS of the entire cohort was 55 months. In a subgroup of 41 nonpancreatic GEP-NETs, efficacy results showed $22 \%$ PR, $17.1 \% \mathrm{MR}, 48.8 \% \mathrm{SD}$, and $12.2 \%$ PD. Relevant but transient myelosuppression (Grade 3/4; CTCAE 3.0) was reported in less than $10 \%$ of cases. No cases of irreversible toxicity, including renal toxicity (Grade 3/4), were reported.

In the past few years, our group in Meldola (Italy) has published a number of papers on Phase II studies focusing on the activity and safety profile of patients treated with $177 \mathrm{Lu}-$ Dotatate. ${ }^{23}$ We demonstrated that, as expected, 18-Fluorodeoxyglucose positron emission tomography (FDG PET) was positive in $67 \%$ of G2 NET cases, but it was also positive in a large portion of G1 cases (54\%). Furthermore, in the same study, FDG PET was found to be an important predictive and prognostic factor. In fact, it was more effective than Ki67 in identifying patients who would respond to PRRT and have a better median PFS. These findings obviously need to be confirmed in larger studies but offer a new and theoretically important option for NET patient stratification.

Our group also carried out a Phase II study of PRRT with 177Lu-Dotatate in patients with advanced, well-differentiated (G1-G2) gastrointestinal (GI)-NETs. ${ }^{24}$ Forty-three patients with radiological PD and a positive Octreoscan ${ }^{\circledR}$ (Mallinckrodt, Inc., Dublin, Republic of Ireland) or 68Ga Dota-peptide PET completed treatment and received a cumulative activity of 18.5 or $27.8 \mathrm{GBq}$ in five cycles according to baseline kidney function, reduction in bone marrow reserve, or effects of previous therapies, all defined as risk factors. Twenty-five (58\%) patients received median full dosage of $25.7 \mathrm{GBq}$ (range 22.2-27.8), while the remaining 18 patients (42\%) received a mean reduced dosage of $18.4 \mathrm{GBq}$ (range 14.4-20.4). According to SWOG criteria, the overall response was a complete response in $7 \%$ of cases and SD in 77\%, with a disease control rate (DCR) of $84 \%$. Median response duration was 25 months (range 7-50). Median PFS was 36 months, while median OS has not yet been reached. Once again, none of the patients showed side effects after either of the Lu-PRRT dosages.

177Lu-Dotatoc has been used in a number of important centers in the last few years, with a promising efficacy and toxicity profile. A recent paper by Baum et $\mathrm{al}^{25}$ reported efficacy and safety data of $177 \mathrm{Lu}$-Dotatoc administered to 56 patients with metastatic and progressive NETs (50\% GI tract, $26.8 \%$ pancreas, and $23.2 \%$ other primary sites). Median dosage was 7.0 GBq every 3 months (mean 2.1 cycles; range 1-4). Efficacy evaluation was performed using computed tomography and/or magnetic resonance imaging according to Response Evaluation Criteria in Solid Tumors 1.1 criteria, and the results were stratified according to the number of administered cycles and the primary tumor origin. The overall median DCR was $93.8 \%$ for patients who received more than one cycle, while median PFS and OS were 17.4 and 34.2 months, respectively, assessed over a follow-up time (mean \pm standard deviation) of $16.1 \pm 12.4$ months. Considering only the GEP-NET patients who received more than one cycle, DCR increased to $100 \%$, while PFS was significantly higher for non-GEP-NET patients (34.5 months non-GEP-NET vs 11.9 months GEP-NET). Moreover, no serious adverse events were observed. One case of selflimiting Grade 3 myelotoxicity was reported and there were no cases of renal toxicity after treatment, even in patients with mild renal insufficiency at baseline.

\section{PRRT retreatment in GEP-NET patients, a new therapeutic application (personal experience)}

The majority of patients with GEP-NETs who relapse after therapy in the early stages of disease still have a good prognosis given the fairly indolent behavior of well-/moderately differentiated neuroendocrine tumors. At our institute, the task of choosing between the many therapeutic options available is the responsibility of a dedicated and experienced multidisciplinary team whose decision is based on the patient's medical history, current clinical conditions, and pathological stage of the disease. In this complex situation, PRRT retreatment is considered for patients who have 
Table 2 Results of our experience of PRRT retreatment

\begin{tabular}{lll}
\hline Evaluation criteria & 90Y-PRRT treatment & I 77Lu-PRRT retreatment \\
\hline Number of patients & 26 & 26 \\
Median activity (range) & $10.8 \mathrm{GBq}(5-14.9)$ & $16.5 \mathrm{GBq}(5.5-22.2)$ \\
Median number of cycles & $5(2-7)$ & $5(2-5)$ \\
$\quad$ CR & 1 & 1 \\
PR & 3 & 1 \\
SD & 22 & 20 \\
PD & $\mathrm{NA}$ & 4 \\
Median PFS, months $(95 \% \mathrm{Cl})$ & $28(20-36)$ & $22(16-\mathrm{nr})$ \\
Median OS, months $(95 \% \mathrm{Cl})$ & & $36(4-70)$ \\
\hline
\end{tabular}

Note: (C) Springer-Verlag Berlin Heidelberg 2015. Severi S, Sansovini M, lanniello A, et al. Feasibility and utility of re-treatment with (I77)Lu-DOTATATE in GEP-NENs relapsed after treatment with (90)Y-DOTATOC. Eur J Nucl Med Mol Imaging. 2015;42(13): 1955-1963. ${ }^{31}$ With permission of Springer.

Abbreviations: CR, complete response; PR, partial response; SD, stable disease; PD, progressive disease; PFS, progression-free survival; OS, overall survival; nr, not reached; $\mathrm{Cl}$, confidence interval; PRRT, peptide receptor radionuclide therapy; NA, not applicable.

received low-dose PRRT as a first treatment and who are still in good general condition, making them amenable to further PRRT. In addition to confirmation of persistent SSTr2 receptor expression with Octreoscan or 68Ga-Dotapeptide PET, patients must have shown a good response to the first treatment (a PFS of at least 12 months is required). The few studies published on this topic are generally based on retrospective data of patients who were not homogeneously treated. In fact, reported experiences vary widely in terms of number of cycles and injected activity in both treatment and retreatment settings. ${ }^{26-30}$

Our institute carried out a Phase II study ${ }^{31}$ to evaluate the feasibility and potential activity of retreatment with lowdosage 177Lu-Dotatate in patients previously treated with 90Y-Dotatoc. Patients were administered a dosage of $500 \mathrm{mCi}$ $(18.5 \mathrm{GBq})$ in five cycles performed 8 weeks apart, with the aim of not exceeding the maximum tolerable doses to the kidneys and bone marrow. The activity was selected on the basis of results from a previous Phase II trial of GEP-NET patients conducted by our team, which did not highlight a statistical difference in terms of DCR between high- and low-dosage groups. The findings prompted us to propose the low dosage for the 177Lu-Dotatate retreatment PRRT protocol. ${ }^{24}$

We enrolled 26 consecutive patients previously treated with 90Y-Dotatoc and with preserved kidney and hematological parameters. Toxicity, DCR, PFS, and prognostic factors were evaluated as primary and secondary objectives. The median administered activity delivered in five cycles was 16.5 GBq. Only two cases of Grade 2 and one case of Grade 3 bone marrow toxicity were observed. One patient experienced Grade 2 renal toxicity, while another, who had previous renal problems, experienced transient Grade 3 toxicity. The DCR was $84.6 \%$ and median PFS was 22 months (95\% confidence interval, 16 - not reached) compared to 28 months (95\% confidence interval, 20-36) after the first 90Y-Dotatoc treatment. The most important prognostic factors were tumor burden and the number of liver metastases (Table 2). ${ }^{31}$

Despite the limited number of patients involved in our study, results suggest that retreatment with low-dosage 177Lu-Dotatate can be considered as a valid therapeutic option for GEP-NET patients who relapse after previous 90Y-Dotatoc treatment (Figure 2). Obviously this kind of advanced patient represents a complex problem given the possible toxic effect of previous PRRT and concurrent therapies. Thus, all therapeutic options must be carefully examined by a multidisciplinary team of experts in the area of neuroendocrine tumors in order to offer patients the best chance of a good clinical response and longer survival.

\section{Therapeutic strategy}

In recent years, PRRT with either 177Lu-Dotatate or 90YDotatoc has been shown to be highly effective and seldom toxic in progressive GI-NETs, generally obtaining results largely comparable with competitive therapies, especially in terms of PFS. Up to now, the burning issue in PRRT treatment has been whether to use 177Lu-Dotatate or $90 \mathrm{Y}$ Dotatoc. We have been accustomed to evaluate differences in terms of radionuclide emission energy and peptide receptor affinity. The dosimetric implications related to lesion volume and possible kidney or bone marrow toxicity are certainly important but are not the only variables to consider.

Recent studies have shown that clinical risk factors such as hypertension, diabetes, or renal or bone marrow impairment caused by previous chemotherapy increase PRRT toxicity, and efforts have been made to identify the minimum effective activity that can make PRRT feasible for high-risk patients as well. At the same time, dosimetric studies have demonstrated that, together with the total administered activity, the total number of cycles also plays an important role in PRRT toxicity. ${ }^{17,19,32}$ In particular, it has been seen that a 
A

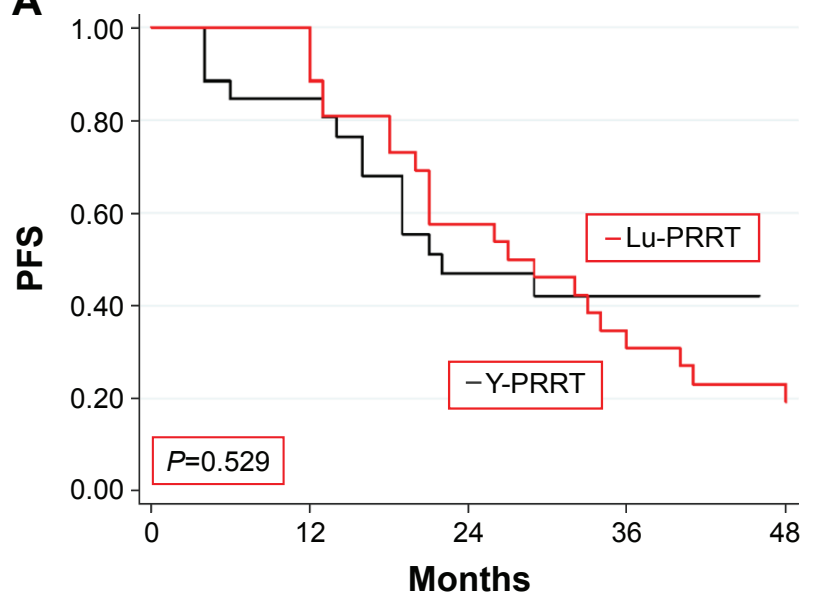

B

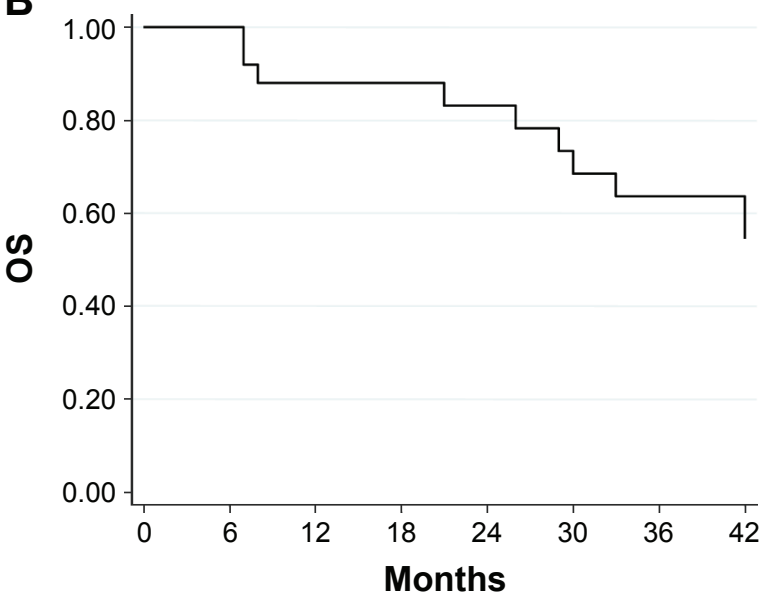

Figure 2 (A) PFS curves of the same group of patients after treatment with 90Y-Dotatoc and after retreatment with low-dose I77Lu-Dotatate. (B) OS curve for retreatment group.

Note: It was not possible to elaborate a posttreatment OS curve as all of the patients included in the study were originally treated in different centers in Italy.

Abbreviations: PFS, progression-free survival; OS, overall survival; 90Y-Dotatoc, 90Y-DOTA-Tyr3-octreotide; 177Lu-Dotatate, I77Lu-DOTA-Tyr3-octreotate.

higher number of cycles with the same cumulative activity protects the kidneys and bone marrow from excess radiation exposure.

There is also an evidence that higher tumor grade, FDG avidity, disease extension, and liver involvement, all considered indicators of disease aggressiveness or prognostic factors, are important aspects to be evaluated before administering PRRT. In fact, nuclear medicine physicians can improve PRRT efficacy in high-risk patients by increasing the total administered activity or the number or frequency of PRRT cycles, or by associating radiosensitizer agents such as capecitabine or capecitabine plus temozolomide, as described by the Australian group. ${ }^{33}$ These new therapeutic options currently under consideration in several centers offer the opportunity of further customizing treatment by increasing dose intensity only when required. There is also a growing interest in studies on liquid biopsy focusing on the prognostic value of circulating agents such as RNA transcripts ${ }^{34}$ or miRNA. The results of these studies will probably provide new and important parameters to increase PRRT effectiveness.

\section{Acknowledgments}

The authors thank Gráinne Tierney and Laura Lotito for editorial assistance.

\section{Disclosure}

The authors report no conflicts of interest in this work.

\section{References}

1. Rindi G. The ENRTS guidelines: the new TNM classification system. Tumori. 2010;96(5):806-809.

2. Lüttges J. What's new? The 2010 WHO classification for tumours of the pancreas. Pathologe. 2011;32 (Suppl 2):332-336.
3. Reubi JC, Schär JC, Waser B, et al. Affinity profiles for human somatostatin receptor subtypes SST1-SST5 of somatostatin radiotracers selected for scintigraphic and radiotherapeutic use. Eur J Nucl Med. 2000;27(3): 273-282.

4. Virgolini I, Ambrosini V, Bomanji JB, et al. Procedure guidelines for PET/CT tumour imaging with 68Ga-DOTA-conjugated peptides: 68GaDOTA-TOC, 68Ga-DOTA-NOC, 68Ga-DOTA-TATE. Eur J Nucl Med Mol Imaging. 2010;37(10):2004-2010.

5. van Essen M, Sundin A, Krenning EP, Kwekkeboom DJ. Neuroendocrine tumours: the role of imaging for diagnosis and therapy. Nat Rev Endocrinol. 2014;10(2):102-114.

6. Valkema R, De Jong M, Bakker WH, et al. Phase I study of peptide receptor radionuclide therapy with [In-DTPA] octreotide: the Rotterdam experience. Semin Nucl Med. 2002;32(2):110-122.

7. Imhof A, Brunner P, Marincek N, et al. Response, survival, and longterm toxicity after therapy with the radiolabeled somatostatin analogue [90Y-DOTA]-TOC in metastasized neuroendocrine cancers. J Clin Oncol. 2011;29(17):2416-2423.

8. Bushnell DL Jr, O'Dorisio TM, O'Dorisio MS, et al. 90Y-edotreotide for metastatic carcinoid refractory to octreotide. J Clin Oncol. 2010;28(10):1652-1659.

9. Bodei L, Cremonesi M, Grana CM, et al. Yttrium-labelled peptides for therapy of NET. Eur J Nucl Med Mol Imaging. 2012;39 (Suppl 1): S93-S102.

10. Bodei L, Cremonesi M, Grana CM, et al. Peptide receptor radionuclide therapy with ${ }^{177} \mathrm{Lu}$-DOTATATE: the IEO phase I-II study. Eur J Nucl Med Mol Imaging. 2011;38(12):2125-2135.

11. Hörsch D, Ezziddin S, Haug A, et al. Peptide receptor radionuclide therapy for neuroendocrine tumors in Germany: first results of a multi-institutional cancer registry. Recent Results Cancer Res. 2013; 194:457-465.

12. Cremonesi M, Ferrari M, Di Dia A, et al. Recent issues on dosimetry and radiobiology for peptide receptor radionuclide therapy. $Q \mathrm{~J} \mathrm{Nucl}$ Med Mol Imaging. 2011;55(2):155-167.

13. Jiménez-Fonseca P, Carmona-Bayonas A, Martín-Pérez E, et al. Health-related quality of life in well-differentiated metastatic gastroenteropancreatic neuroendocrine tumors. Cancer Metastasis Rev. 2015; 34(3):381-400

14. Bodei L, Mueller-Brand J, Baum RP, et al. The joint IAEA, EANM, and SNMMI practical guidance on peptide receptor radionuclide therapy (PRRNT) in neuroendocrine tumours. Eur J Nucl Med Mol Imaging. 2013;40(5):800-816.

15. de Jong M, Krenning E. New advances in peptide receptor radionuclide therapy. J Nucl Med. 2002;43(5):617-620. 
16. Bernard BF, Krenning EP, Breeman WA, et al. D-lysine reduction of indium-111 octreotide and yttrium-90 octreotide renal uptake. $\mathrm{J} \mathrm{Nucl}$ Med. 1997;38(12):1929-1933.

17. Dale R. Use of the linear-quadratic radiobiological model for quantifying kidney response in targeted radiotherapy. Cancer Biother Radiopharm. 2004;19(3):363-370.

18. Barone R, Borson-Chazot F, Valkema R, et al. Patient specific dosimetry in predicting renal toxicity with (90)Y-DOTATOC: relevance of kidney volume and dose rate in finding a dose-effect relationship. $J \mathrm{Nucl} \mathrm{Med}$. 2005;46 (Suppl 1):99S-106S.

19. Bodei L, Cremonesi M, Ferrari M, et al. Long-term evaluation of renal toxicity after peptide receptor radionuclide therapy with $90 \mathrm{Y}$ DOTATOC and 177Lu-DOTATATE: the role of associated risk factors. Eur J Nucl Med Mol Imaging. 2008;35(10):1847-1856.

20. Valkema R, Pauwels S, Kvols LK, et al. Survival and responsafter peptide receptor radionuclide therapy with [90Y-DOTA0, Tyr3] octreotide in patients with advanced gastroenteropancreatic neuroendocrinetumors. Semin Nucl Med. 2006;36(2):147-156.

21. Kwekkeboom DJ, de Herder WW, Kam BL, et al. Treatment with the radiolabeled somatostatin analog [177 Lu-DOTA 0, Tyr3]octreotate: toxicity, efficacy, and survival. J Clin Oncol. 2008;26(13):2124-2130.

22. Ezziddin S, Attassi M, Yong-Hing CJ, et al. Predictors of long-term outcome in patients with well-differentiated gastroenteropancreatic neuroendocrine tumors after peptide receptor radionuclide therapy with 177Lu-octreotate. J Nucl Med. 2014;55(2):183-190.

23. Severi S, Nanni O, Bodei L, et al. Role of 18 FDG PET/CT in patients treated with $177 \mathrm{Lu}-\mathrm{DOTATATE}$ for advanced differentiated neuroendocrine tumours. Eur J Nucl Med Mol Imaging. 2013;40(6):881-888.

24. Paganelli G, Sansovini M, Ambrosetti A, et al. 177 Lu-Dota-octreotate radionuclide therapy of advanced gastrointestinal neuroendocrine tumors: results from a phase II study. Eur J Nucl Med Mol Imaging. 2014;41:1845-1851.

25. Baum RP, Kluge AW, Kulkarni H, et al. [(177)Lu-DOTA](0)-D-Phe(1)Tyr(3)-Octreotide ((177)Lu-DOTATOC) For peptide receptor radiotherapy in patients with advanced neuroendocrine tumours: a phase-II study. Theranostics. 2016;6(4):501-510.

26. Forrer F, Uusijärvi H, Storch D, Maecke HR, Mueller-Brand J. Treatment with 177Lu-DOTATOC of patients with relapse of neuroendocrine tumors after treatment with 90Y-DOTATOC. J Nucl Med. 2005; 46:1310-1316.

27. Pach D, Sowa-Staszczak A, Kunikowska J, et al. Repeated cycles of peptide receptor radionuclide therapy (PRRT)-results and side-effects of the radioisotope $90 \mathrm{Y}$-DOTA TATE, 177Lu-DOTA TATE or 90Y/177Lu-DOTA TATE therapy in patients with disseminated NET. Radiother Oncol. 2012;102(1):45-50.
28. van Essen M, Krenning EP, Kam BL, de Herder W, Feelders RA, Kwekkeboom DJ. Salvage therapy with 177Lu-octreotate in patients with bronchial and gastroenteropancreatic neuroendocrine tumors. J Nucl Med. 2010;51(3):383-390.

29. Sabet A, Haslerud T, Pape UF, et al. Outcome and toxicity of salvage therapy with $177 \mathrm{Lu}$-octreotate in patients with metastatic gastroenteropancreatic neuroendocrine tumours. Eur J Nucl Med Mol Imaging. 2014;41(2):205-210.

30. Prasad V, Brenner W, Modlin IM. How smart is peptide receptor radionuclide therapy of neuroendocrine tumors especially in the salvage setting? The clinician's perspective. Eur J Nucl Med Mol Imaging. 2014;41(2):202-204.

31. Severi S, Sansovini M, Ianniello A, et al. Feasibility and utility of re-treatment with 177Lu-DOTATATE in GEP-NENs relapsed after treatment with 90Y-DOTATOC. Eur J Nucl Med Mol Imaging. 2015; 42(13):1955-1963.

32. Sarnelli A, Guerriero F, Botta F, et al. Therapeutic schemes in $177 \mathrm{Lu}$ and 90Y-PRRT: radiobiological considerations. $Q \mathrm{~J} \mathrm{Nucl} \mathrm{Med} \mathrm{Mol}$ Imaging. Epub November 17, 2015.

33. Kesavan M, Claringbold PG, Harvey Turner J. Hematological toxicity of combined 177Lu radiopeptide chemotherapy of gastroenteropancreatic neuroendocrine tumors in long term follow up. Neuroendocrinology. 2014;99(2):108-117.

34. Bodei L, Kidd M, Modlin IM, et al. Measurement of circulating transcripts and gene cluster analysis predicts and defines therapeutic efficacy of peptide receptor radionuclide therapy (PRRT) in neuroendocrine tumors. Eur J Nucl Med Mol Imaging. 2016;43(5):839-851.

35. Garkavij M, Nickel M, Sjorgreen-Gleisner K, et al. 177Lu[DOTA0,Tyr3] octreotate therapy in patients with disseminated neuroendrocrine tumors: Analysis of dosimetry with impact on future therapeutic strategy. Cancer. 2010;116(4 Suppl):1084-1092.

36. Swärd C, Bernhardt P, Ahlman H, et al. [177Lu-DOTA 0-Tyr 3]-octreotate treatment in patients with disseminated gastroenteropancreatic neuroendocrine tumors: the value of measuring absorbed dose to the kidney. World J Surg. 2010;34(6):1368-1372.

37. Bodei L, Cremonesi M, Grana CM, et al. Peptide receptor radionuclide therapy with ${ }^{177} \mathrm{Lu}$-DOTATATE: the IEO phase I-II study. Eur J Nucl Med Mol Imaging. 2011;38(12):2125-2135
OncoTargets and Therapy

\section{Publish your work in this journal}

OncoTargets and Therapy is an international, peer-reviewed, open access journal focusing on the pathological basis of all cancers, potential targets for therapy and treatment protocols employed to improve the management of cancer patients. The journal also focuses on the impact of management programs and new therapeutic agents and protocols on

\section{Dovepress}

patient perspectives such as quality of life, adherence and satisfaction. The manuscript management system is completely online and includes a very quick and fair peer-review system, which is all easy to use. Visit http://www.dovepress.com/testimonials.php to read real quotes from published authors. 